\title{
Effects of the Degree of Substitution on the Properties and Structures of Side Chain Liquid Crystalline Polysiloxanes
}

\author{
Koichiro Yonetake, ${ }^{*}$ Masaki NaKagomi, Mitsuru Ueda, \\ and Toru Masuko \\ Faculty of Engineering, Yamagata University, \\ Jonan 4, Yonezawa, Yamagata 992, Japan
}

(Received August 1, 1996)

\begin{abstract}
Poly[6-(4-cyanobiphenyl-4'-oxy)-hexylmetylsiloxanes] (PCS-n) with various degrees of substitution (DS) were prepared, and the effects of the degree of substitution on the phase transition, structures, and liquid crystallization of PCS-n were investigated. PCS-n showed $S_{\mathrm{Ad}}$ in the temperature range from glass transition temperature $\left(T_{\mathrm{g}}\right)$ to clearing temperature $\left(T_{\mathrm{i}}\right)$ in spite of various DS. $T_{\mathrm{g}}$ and $T_{\mathrm{i}}$ increased linearly with DS, and the temperature range of $S_{\mathrm{A}}$ phase between $T_{\mathrm{g}}$ and $T_{\mathrm{i}}$ expanded with DS. The layer spacing $d_{\mathrm{L}}$ of the $S_{\mathrm{A}}$ structure decreased with increasing DS, since the non-mesogenic flexible backbones were squeezed between smectic layers. $d_{\mathbf{L}}$ of the sample with lower DS highly decreased with increasing temperature, due to entropy relaxation of backbones between the $S_{\mathrm{A}}$ layers. DS remarkably influenced the liquid crystallization of PCS-n. PCS-n with higher DS exhibited faster growth rates of liquid-crystalline textures compared with lower DS. KEY WORDS Side-Chain Liquid-Crystalline Polymer / Degree of Substitution / Smectic A / Polysiloxane / Liquid Crystallization /
\end{abstract}

The synthesis and characterization of liquid crystalline polymers have become of interest in recent years, because of potential uses in electrooptical devices. ${ }^{1-6}$ Polysiloxanes have several advantages over other types of polymers due to lower glass transition temperatures and great flexibility of the backbones. ${ }^{5}$

Generally, the phase transition behavior, liquid crystalline textures, and stability of mesophase depend on chemical structures of mesogenic groups, length of flexible spacer group, and mesogenic terminal groups. Many polysiloxanes with different mesogenic side chain units have been synthesized and the influence of molecular weight, nature of mesogenic group, length of spacer group, and composition of copolymers with different mesogens on the liquid crystalline property have been investigated. ${ }^{1,2}$

The amount of substitution of mesogenic side chain influences the phase transition and liquid crystalline properties. ${ }^{7-12}$ Percec et al. ${ }^{8,9}$ investigated the liquidcrystalline properties of poly(methylsiloxane-co-dimethyl-siloxane)s containing 2-[4-(2(S)-methyl-1-butoxy)phenyl]-5-(11-undecanyl)-1,3,2-dioxaborinane side groups. The phase transition temperatures of the copolymers increased with composition of mesogenic structural unit. ${ }^{9}$ Diele et al. ${ }^{11}$ explored the dependence of amount of substitution on liquid crystalline structure by means of X-ray diffraction analysis. They suggest that the smectic monolayer thickness remarkably depend on the amount of substitution and a flexible main chain was forced to take part in the layer formation, due to the domaining interaction of the mesogenic groups. Percec et al. ${ }^{10}$ reported that the thickness of the smectic layer of above copolymer increased with decrease of structural units containing mesogenic units. Thus, the flexible backbone is squeezed between smectic layers in the side-chain liquid-crystalline polysiloxanes. ${ }^{13}$

We investigated the growth of liquid crystal for poly-

$\dagger$ To whom all correspondence should be addressed. siloxane having 6-(4-cyanobiphenyl-4'-oxy)-1-hexene pendant in the isothermal state, and the rates of liquidcrystallization were determined at various liquid-crystallization temperatures. ${ }^{14}$ The growth of the liquid crystal during the liquid-crystallization of the polysiloxane is possible to be influenced by the concentration of the mesogenic structural unit. Little is known about the influence of DS on the liquid-crystallization of polysiloxanes. In the present work, we prepared side-chain liquid-crystalline polysiloxanes with various DS of 4cyano-4'-hydroxybiphenyl mesogenic group and investigated the influence of DS on the phase transition, structures, and liquid-crystallization on the basis of thermal analysis, optical microscope, and X-ray diffraction method.

\section{EXPERIMENTAL}

\section{Synthesis of Liquid Crystalline Polysiloxanes}

The mesogenic compound 6-(4-cyanobiphenyl-4'oxy)-1-hexene was prepared by the condensation of 4'hydroxy-4-cyanobiphenyl with 6-bromo-1-hexene. Liquid crystalline polysiloxanes with various DS were prepared by hydrosilylation between poly(methylsiloxane) and 6-(4-cyanobiphenyl-4'-oxy)-1-hexene in the presence of platinum catalyst. Poly(methylsiloxane) having $\bar{M}_{n}=$ $3,800, \bar{M}_{w}=10,500, \bar{M}_{w} / \bar{M}_{n}=2.7$ was supplied by Chisso Co. Synthesized polymers, poly[6-(4-cyanobiphenyl-4'oxy)-hexylmetylsiloxane] (PCS), were purified by repeated precipitation from methylene chloride solution with excess methanol, and then were isolated by the freeze-dry treatment.

Polymers were defined as corresponding polysiloxanes containing the 6(4-cyanobiphenyl-4'-oxy)hexyl group as a side chain mesogenic unit, by IR spectroscopy, ${ }^{1} \mathrm{H}$ NMR, and ${ }^{13} \mathrm{C}$ NMR. The IR spectra exhibited characteristic the $\mathrm{C} \equiv \mathrm{N}$ bond stretching at $2220 \mathrm{~cm}^{-1}$, the aromatic $\mathrm{C}=\mathrm{C}$ bond stretching at 1604 and $1495 \mathrm{~cm}^{-1}$, and $\mathrm{C}-\mathrm{O}-\mathrm{C}$ bond stretching at 1253 and $1020 \mathrm{~cm}^{-1}$. The 
${ }^{1} \mathrm{H}$ NMR (JEOL EX $\left.270(270 \mathrm{MHz})\right)$ spectrum of the polymer consists of multiplets for the aromatic protons $(\delta=6.8-7.7 \mathrm{ppm}, 8 \mathrm{H})$, a singlet for the silyl hydride $(\delta=4.75 \mathrm{ppm})$, several broad peaks for methylene protons adjacent to ether group $(\delta=3.9 \mathrm{ppm}, 2 \mathrm{H})$, to $\mathrm{Si}$ atom $(\delta=0.6 \mathrm{ppm}, 2 \mathrm{H})$ and other methylene protons $(\delta=1.4 \mathrm{ppm}, 6 \mathrm{H})$, and methyl protons $(\delta=0.1 \mathrm{ppm}, 3 \mathrm{H})$, respectively. The most conclusive spectra evidence for the proposed polymer structures was provided by ${ }^{13} \mathrm{C}$ NMR (JEOL EX 270). The calculated chemical shifts are all within $3 \mathrm{ppm}$ of observed values. No duplication peaks was found, clearly indicating formation of the expected polymers.

DS was obtained by the ratio of the integration of silyl hydride of polysiloxane to aromatic protons of the mesogen unit. DS of polymers were $34,43,59,66,70$, and $83 \%$. These polymer samples were coded as PCS-n, where the number $\mathrm{n}$ denotes the DS.

\section{Thermal Analysis}

Differential scanning calorimetry (DSC) of the samples was performed by DSC 200 (Seiko I \& E Ltd.) under an $\mathrm{N}_{2}$ purge; heating and cooling rates were $20^{\circ} \mathrm{C} \mathrm{min}^{-1}$ and $10^{\circ} \mathrm{C} \min ^{-1}$, respectively.

\section{Polarizing Optical Microscopy}

Changes in optical textures of the samples during heating and cooling processes were examined using the polarizing optical microscope (POM) equipped with a hot stage (Linkam Co., TH-600RMS) under an $\mathrm{N}_{2}$ purge. Growth of liquid crystalline textures was observed under POM with a videocassette recorder system. ${ }^{14}$

\section{$X$-Ray Diffraction Measurements}

X-Ray diffraction experiments were carried out by a RAD-rA diffractometer (Rigaku Denki Co., Ltd.) equipped with a heating device with temperature accuracy of $\pm 0.2^{\circ} \mathrm{C}$. Nickel-filtered $\mathrm{Cu}-K_{\alpha}$ radiation was employed. Changes in the X-ray scattering (XRS) traces during heating and cooling processes were recorded by a scintillation counter system with a $1.0 \mathrm{~mm}$ diameter pin-hole collimator and $1 \times 1^{\circ}$ receiving slit. Diffractometry was performed in transmission. XRS traces were obtained by a step-scanning method: step width and fixed time were programmed for steps of 0.05 deg every $4 \mathrm{~s}$. When $d$-spacings of the reflections were measured, the step and fixed time were programmed for an angle of $0.01 \mathrm{deg}$ and a $10 \mathrm{~s}$ step, respectively. The XRS photographs were taken by a flat Laue camera with a $0.5 \mathrm{~mm}$ diameter pin-hole collimator.

\section{RESULTS AND DISCUSSION}

\section{Influence of the Degree of Substitution on Liquid Crystal-} line Properties

DSC traces of the liquid crystalline polysiloxanes with different DS are illustrated in Figure 1, which correspond to the 2 nd-heating process. Marked glass transition $\left(T_{\mathrm{g}}\right)$ and single endothermic peak were observed on the DSC trace of each sample. The endothermic peak of each sample was assigned to be a clearing temperature $\left(T_{\mathrm{i}}\right)$, as a dark field appeared above the endothermic peak under POM. After cooling from the isotropic melt,

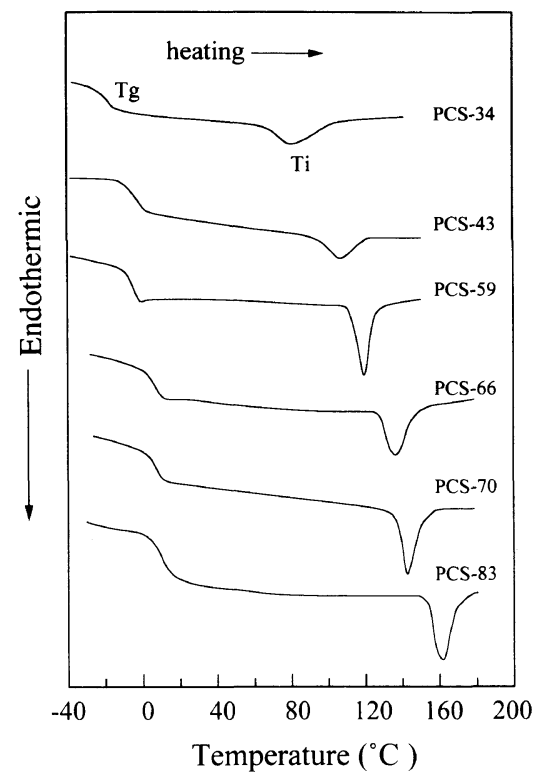

Figure 1. DSC traces of PCS-n during 2nd-heating process.

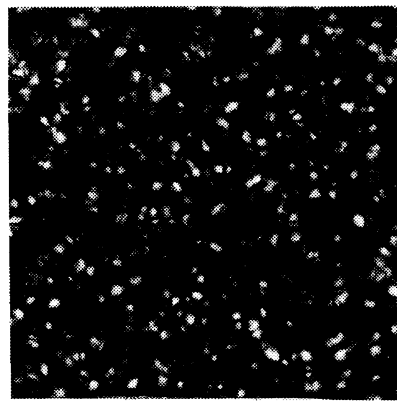

(a) PCS-34

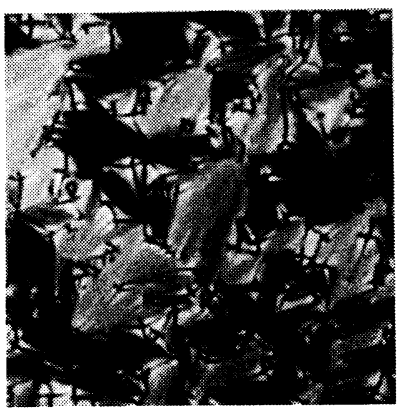

(c) PCS-66

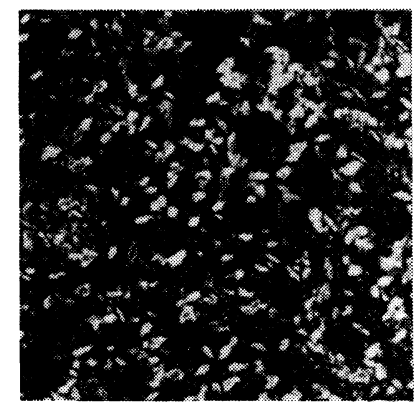

(b) PCS-59

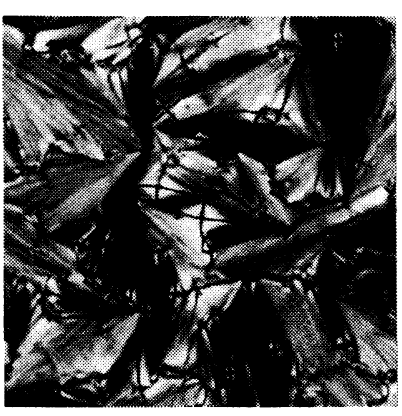

(d) PCS-83

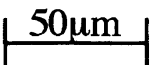

Figure 2. Polarizing Optical micrographs of PCS-34 (a), PCS-59 (b), PCS-66 (c), and PCS-83 (d) taken at room temperature.

well-defined focal-conic fan texture formed in the samples with higher DS, as shown in Figure 2. In samples with lower DS, optical textures were very small; micro-grain liquid-crystalline textures formed.

As shown in Figure 3, the XRS patterns of oriented samples for PCS-34, PCS-59, and PCS-83 indicate a diffuse meridional reflection at a wide-angle area, and periodic equatorial arcs at a small-angle area, characteristic of smectic A $\left(S_{\mathrm{A}}\right)$ phase. Other samples with different DS showed similar XRS patterns. Thus, the PCS-n exhibited $S_{\mathrm{A}}$ phase below $T_{\mathrm{i}}$ whatever DS was. The values of the layer spacing of $S_{\mathrm{A}}$ determined from the reflection 


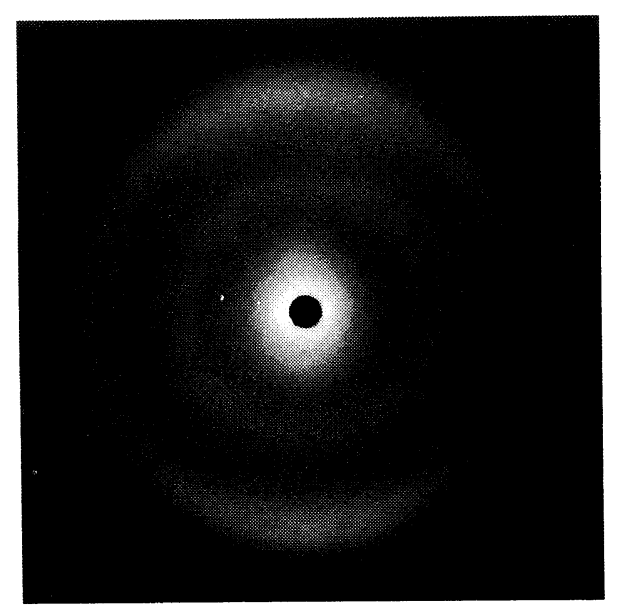

(a) PCS-34
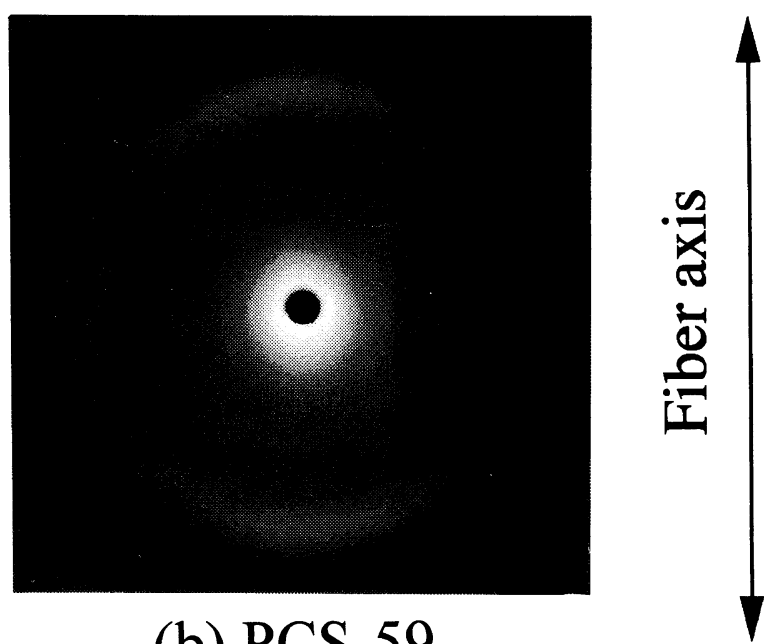

(b) PCS-59

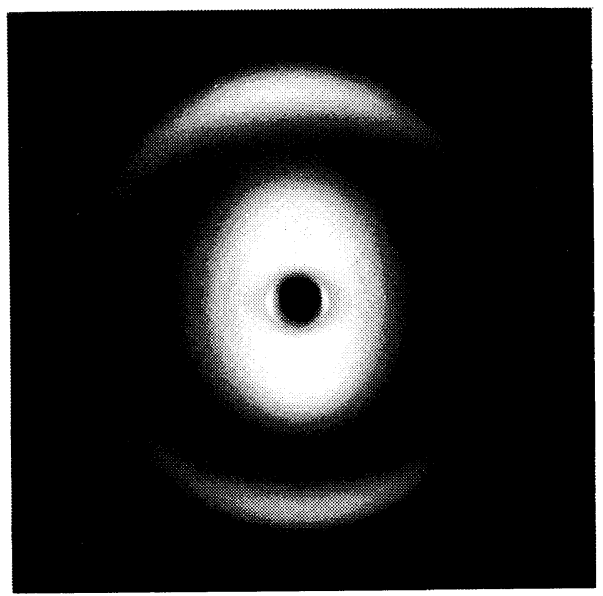

(c) PCS-83

Figure 3. XRD patterns of oriented samples for PCS-34 (a), PCS-59 (b), and PCS-83 (c) taken at room temperature.

at the small-angle area are approximately $1.6-1.9$ times those of the side chain $(1.94 \mathrm{~nm})$. This shows the formation of an interdigitated bilayer structure $\left(S_{\mathrm{Ad}}\right)^{15}$ because of interactions of strong dipoles induced in the mesogenic groups by $\mathrm{CN}$ substituents. ${ }^{16,17}$

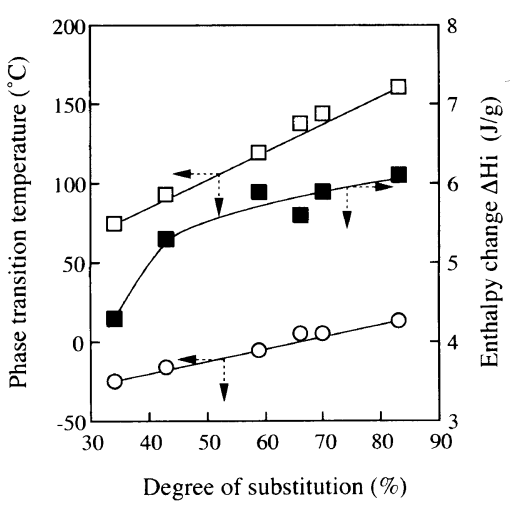

Figure 4. Changes in phase transition temperature $\left(T_{\mathrm{g}}, T_{\mathrm{i}}\right)$ and enthlpy change $\left(\Delta H_{\mathrm{m}}\right)$ of PCS-n against degree of substitution. $\bigcirc, T_{\mathrm{g}}$, $\square, T_{\mathrm{i}}, \square, \Delta H_{\mathrm{i}}$.

The phase transition temperatures of these samples are plotted against DS in Figure 4. Temperatures of $T_{\mathrm{g}}$ and $T_{\mathrm{i}}$ increased linearly with DS. The tendency of phase transition temperature is similar to the results of the copolysiloxanes containing 2-[4-(2(S)-methyl-1-butoxy)phenyl]-5-(11-undecanyl)-1,3,2-dioxaborinane side groups. ${ }^{9}$ The slope of the line for $T_{\mathrm{i}}$ was larger than that for $T_{\mathrm{g}}$. Thus, the temperature range of $S_{\mathrm{A}}$ phase between $T_{\mathrm{g}}$ and $T_{\mathrm{i}}$ expanded with DS; the stability of $S_{\mathrm{A}}$ phase was enhanced by increase in DS. Generally, side groups have profound effect on $T_{\mathrm{g}}$; large and bulky side groups cause great stiffening. The increase in $T_{\mathrm{g}}$ comes from steric hindrance by increase in the large and bulky mesogenic side chains. In other words, lowing of $T_{\mathrm{g}}$ with decreasing substitution is due to diluting the mesogenic groups along the backbone with non-mesogenic segments. ${ }^{18}$ The enthalpy change $\left(\Delta H_{\mathrm{i}}\right)$ estimated from the endothermic peak increased with DS, as shown in Figure 4. Thus, $S_{\mathrm{A}}$ phase of PCS-n may possibly be thermodynamically stable with increasing DS.

\section{Smectic Layer Structures of PCS-n}

XRS intensity curves were measured to investigate the order of the layer structures. XRS traces are illustrated in Figure 5. The periodic reflection associated with layer structure of $S_{\mathrm{Ad}}$ was observed in the small angle area in each sample. High order reflections appeared in the samples with higher DS. 2nd and 3rd order reflections could be obtained clearly in the case of PCS-83; $d$-spacings of these three Bragg maxima $\left(d_{1}, d_{2}\right.$, and $\left.d_{3}\right)$ were determined as $3.22 \mathrm{~nm}, 1.63 \mathrm{~nm}$, and $1.08 \mathrm{~nm}$, respectively. The higher order reflections disappeared in PCS-43 and PCS-34. Accordingly the order of $S_{\mathrm{A}}$ layer structure was governed by DS. These findings show that the thermal stability of $S_{\mathrm{A}}$ phase is enhanced by increase in DS.

The diffuse arc set lied on the meridian with a spacing of approximately $0.44 \mathrm{~nm}$, associated with intermolecular spacing $\left(d_{\mathrm{m}}\right)$; that is, the distance between successive mesogenic side groups. $d_{\mathrm{m}}$ almost remained unchanged with increasing DS, as shown in Figure 6. Thus, the interlayer packing mode of $S_{\text {Ad }}$ for PCS-n is independent of DS. One reason for this is that the $S_{\mathrm{Ad}}$ phase is formed by interactions of strong dipoles induced in the mesogenic groups by $\mathrm{CN}$ substituents. The layer spacing $\left(d_{\mathrm{L}}\right)$ determined from the low angle reflection $\left(d_{1}\right)$ decreased with increasing DS, as shown in Figure 6. The decrease in $d_{\mathbf{L}}$ 


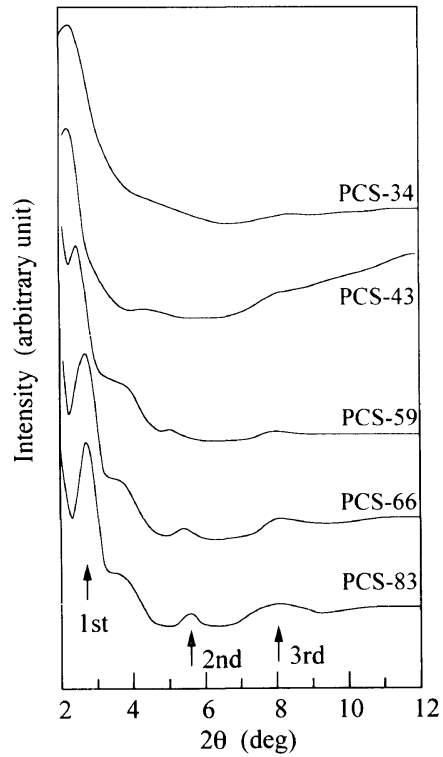

Figure 5. Changes in XRD traces of PCS-n with substitution.

is ascribed to decrease in backbones with non-mesogenic segments between smectic layers. In other words, the non-mesogenic flexible backbone is forced to take part in the layer formation of PCS- $n$. This is similar to copolysiloxanes containing 2-[4-(2(S)-methyl-1-butoxy)phenyl]-5-(11-undecanyl)-1,3,2-dioxaborinane side groups. ${ }^{9}$ Chain packing models were proposed in sidechain liquid-liquid crystalline polysiloxanes based on the results of X-ray diffraction studies ${ }^{9,11,13}$ and other sidechain liquid-crystalline polymers. ${ }^{19}$ Thus, it was confirmed that non-mesogenic backbones are confined between smectic layers in the case of PCS-n.

Many non-mesogenic flexible backbones between the smectic layers may influence the order of smectic layer structure and thermodynamical stability. The disappearance of 2 nd or $3 \mathrm{rd}$ order reflections in the low angle area presumably is due to the flexible backbones confined between the layers.

\section{Temperature Dependence of Layer Spacings}

Flexible backbones between the smectic layer may influence the structural change of the smectic layer at elevated temperatures because of large chain mobility. In this section the influence of DS on the smectic layer structure of PCS-n during the heating process was investigated. $d_{\mathrm{L}}$ of PCS-n was measured during the heating process using the XRS method. Changes in $d_{\mathrm{L}}$ of PCS-43, PCS-59, PCS-66, and PCS-83 are plotted as a function of temperature in Figure 7. $d_{\mathrm{L}}$ decreased with temperature, whereas the $d_{\mathrm{L}}$ of PCS-66 and PCS-83 slightly increased with increasing temperature in lower temperature range, and started to decrease at approximately 40 and $55^{\circ} \mathrm{C}$ respectively. Increase in $d_{\mathrm{L}}$ is considered due to thermal expansion of the smectic layers. The onset of temperature decrease in $d_{\mathrm{L}}$ tends to lower with decreasing DS. In the cases of PCS-43 and PCS-59, increase in $d_{\mathrm{L}}$ is presumably observed at lower temperature range close to $T_{\mathrm{g}}$ 's. $d_{\mathrm{m}}$ of each sample increased monotonously with temperature by thermal expansion, so that little change was observed in the interlayer packing mode at the temperature range. These findings suggest that decrease in $d_{\mathrm{L}}$ with increasing temperature is ascribable to the con-

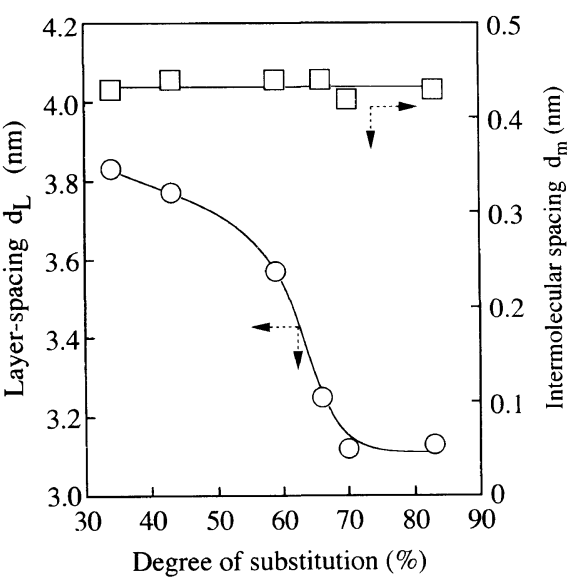

Figure 6. Changes in layer-spacing $\left(d_{\mathrm{L}}\right)$ and intermolecular spacing $\left(d_{\mathrm{m}}\right)$ of PCS-n against degree of substitution. $\bigcirc, d_{1} ; \mathbf{\square}, d_{\mathrm{m}}$.

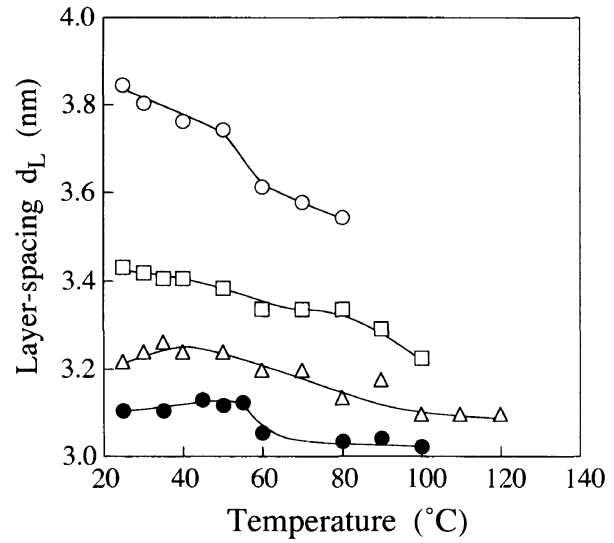

Figure 7. Changes in layer-spacing $d_{\mathrm{L}}$ of PCS against temperature. O, PCS-43; $\square$, PCS-59; $\triangle$, PSC-66; 9 , PCS-83.

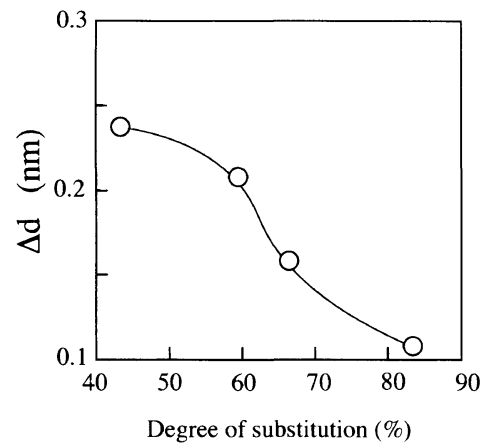

Figure 8. Changes in $\Delta d$ of PCS against degree of substitution. $\Delta d_{\mathrm{m}}=d_{\mathrm{Lmax}}-d_{\mathrm{Lmin}}$ in Figure 7 .

formational change of the flexible spacers and backbones between the smectic layers. Change in $d_{\mathrm{L}}$ was reversible in repeated heating and cooling.

The difference $\left(\Delta d_{\mathrm{L}}\right)$ between maxima and minima of $d_{\mathrm{L}}$ in Figure 7 is plotted against DS in Figure 8. $\Delta d_{\mathrm{L}}$ decreased with DS. The variation in the $d_{\mathrm{L}}$ due to the conformational change of the flexible spacer is considered the same in each sample, as the length of flexible spacer is the same in any sample. From this it follows that change in $\Delta d_{\mathrm{L}}$ with DS is governed by the number of non-mesogenic backbones. This can be understood by the following reason. The backbones might have an internal stress by the formation of $S_{\mathrm{A}}$, because of the $S_{\mathrm{A}}$ layer packing with overlapping antiparallel side groups with strong dipoles induced by $\mathrm{CN}$ substituents. On heating, the internal stress of the backbones is relaxed, 

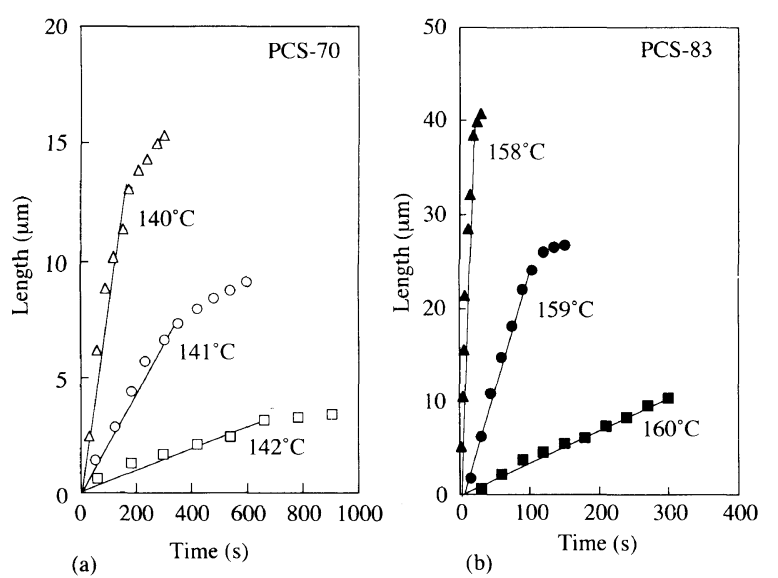

Figure 9. Changes in length of needle-like textures of PCS-70 (a) and PCS-83 (b) against time at various temperatures.

and simultaneously, $d_{\mathrm{L}}$ decrease in comparatively high temperature range. Therefore, the decrease in $d_{\mathbf{L}}$ with increasing temperature results from the entropy relaxation of backbones between the $S_{\mathrm{A}}$ layers. On cooling, $d_{\mathrm{L}}$ increased reversibly by reformation of $S_{\mathrm{A}}$ structure.

\section{Influence of Degree of Substitution on Liquid Crystalliza- tion of PCS-n}

In the case of PCS, thin needle-like textures with $1.8-2.5 \mu \mathrm{m}$ width first appeared at high temperature range close to $T_{\mathrm{i}}$ on cooling from the isotropic phase. On further cooling, the needles developed rapidly to batonnets, and then the batonnets coalesced into the well-defined focal-conic fan texture. The needle-like texture grew preferentially in the direction of the long axis of the needle with time in the isothermal state close to $T_{\mathrm{i}}$. The rates of growth in the liquid crystallization process of PCS- 83 sample were determined in the previous paper. ${ }^{14}$ The growth rates were $163 \times 10^{-2}, 23.5 \times$ $10^{-2}$, and $3.49 \times 10^{-2} \mu \mathrm{m} \mathrm{min}^{-1}$ at 158,159 , and $160^{\circ} \mathrm{C}$, respectively. ${ }^{14}$

The growth of the liquid crystal is possibly influenced by the concentration of the mesogenic structural unit. The growth of needle-like texture also took place at the temperatures close to $T_{\mathrm{i}}\left(144^{\circ} \mathrm{C}\right)$ in PCS-70. In other samples with lower DS, no growth of needle-like texture was observed. Needle-like textures hardly grew in samples with lower DS, when the liquid-crystallization time was prolonged. Thus the layer structures of smectic A in the samples with lower DS exhibited comparatively less order and were thermodinamically unstable because of increased non-mesogenic flexible backbone. The backbones were relaxed at the elevated temperatures as described above.

Figure 9 shows changes in the length of needle-like textures of PCS-70 and PCS-83 with increasing liquid crystallization time. The growth rates for PCS-70 were $6.65 \times 10^{-2}, 2.00 \times 10^{-2}$, and $0.38 \times 10^{-2} \mu \mathrm{m} \mathrm{min}^{-1}$ at 140,141 , and $142^{\circ} \mathrm{C}$, respectively. As the rates of the liquid-crystallization increased with decreasing liquidcrystallization temperature, it was impossible to measure the growth rate at lower temperature. The growth rates of PCS-83 were faster than those of PCS-70. The difference of growth rates between them is due to the concentration of mesogenic side groups, and mobility of backbones and self-diffusion of segments at each liquidcrystallization temperature. Thus the liquid-crystallization of PCS-n remarkably depends on DS.

\section{CONCLUSION}

The influence of the degree of substitution on the phase transition, structures and liquid-crystallization of the side chain liquid crystalline polysiloxane containing 4-cyano4'-hydroxybiphenyl mesogenic group were investigated, and the following findings were obtained.

PCS-n showed $S_{\text {Ad }}$ in the temperature range from $T_{\mathrm{g}}$ to $T_{\mathrm{i}}$ in spite of various DS. $T_{\mathrm{g}}$ and $T_{\mathrm{i}}$ increased linearly with DS, and the temperature range of $S_{\mathrm{A}}$ phase between $T_{\mathrm{g}}$ and $T_{\mathrm{i}}$ expanded with DS. The layer spacing $d_{\mathrm{L}}$ of the $S_{\mathrm{A}}$ structure decreased with increasing DS, since the non-mesogenic flexible backbones were squeezed between the smectic layers. $d_{\mathrm{L}}$ decreased with increasing temperature, and $\Delta d_{\mathrm{L}}$ increased with DS. The decrease in $d_{\mathbf{L}}$ with increasing temperature may thus be due to entropy relaxation of backbones between $S_{\mathrm{A}}$ layers.

Thin needle-like textures first appeared at high temperature range close to $T_{\mathrm{i}}$ on cooling from the isotropic phase in PCS-70 and PCS-83 with comparatively high DS. They grew preferentially in the direction of the long axis. The growth rates of PCS-83 were faster than those of PCS-70. In other samples with lower DS, no growth of needle-like texture was observed. The liquid-crystallization of PCS-n remarkably depended on DS. This results from the concentration of mesogenic side groups, and mobility of the backbones and self-diffusion of the segments.

\section{REFERENCES}

1. H. Finkelmann, M. Happ, M. Portugal, and H. Ringsdorf, Makromol. Chem., 179, 2541 (1978).

2. G. Rehage and H. Finkelmann, Adv. Polym. Sci., 60/61, 99 (1984).

3. V. P. Shibaev and N. A. Plate, Adv. Polym. Sci., 60/61, 173 (1984).

4. N. A. Plate, R. V. Talrose, Y. S. Freidzon, and V. P. Shibaev, Polym. J., 19, 135 (1987).

5. H. Finkelmann, D. Naegele, and H. Ringsdorf, Makromol. Chem., 180, 803 (1979).

6. R. V. Talroze, S. G. Kostromin, V. P. Shibaev, N. A. Plate, H Kresse, K. Sauer, and D. Demus, Polym. J., 2, 305 (1981)

7. C. S. Hsu and V. Percec, Polym. Bull., 18, 91 (1987).

8. B. Hahn and V. Percec, Macromolecules, 20, 1986 (1987).

9. V. Percec and B. Hahn, Macromolecule, 22, 1588 (1989).

10. V. Percec, B. Hahn, M. Ebert, and J. H. Wendorff, Macromolecules, 23, 2029 (1990).

11. S. Diele, S. Oelsner, F. Kuschel, B. Hisgen, H. Ringsdorf, and R. Zentel, Makromol. Chem., 188, 1993 (1987).

12. S. Piercourt, N. Lacoudre, A. L. Borgne, N. Spassky, C. Friedrich, and C. Noel, Makromol. Chem., 193, 705 (1992).

13. V. Percec and D. Tomazos, Polymer, 31, 1658 (1990).

14. K. Yonetake, M. Nakagomi, and T. Masuko, Polym. J., 27, 1157 (1995).

15. C. Noel, in "Liquid Crystal Polymers, A. A. Collyer, Ed., Elsevier Applied Science, London, 1992, pp. 31-101.

16. S. G. Kostromin, V. V. Sinitsyn, R. V. Tal'rose, and V. P Shibayev, Polym. Sci. U.S.S.R., 26, 370 (1984).

17. P. A. Gemmell, G. W. Gray, and D. Lacey, Mol. Cryst. Liq. Cryst., 122, 205 (1985).

18. H. Ringsdorf and A. Schneller, Makromol. Chem. Rapid Commun., 3, 1982 (1982).

19. L. Noirez and G. Pepy, Physica Scripta, T25, 102 (1989). 